\title{
Career Paths of Eminent Researchers in Marriage and Family Therapy
}

Sarah R. Droubay

Thorana S. Nelson

Lee N. Johnson

Brigham Young University - Provo, lee_johnson@byu.edu

Scott A. Ketring

Follow this and additional works at: https://scholarsarchive.byu.edu/facpub

Part of the Family, Life Course, and Society Commons

\section{Original Publication Citation}

Droubay, S. R., Nelson, T. S., Johnson, L. N., \& Ketring, S. A. (2004). Developing a program of research: A content analysis. Contemporary Family Therapy, 26, 503-520.

\section{Recommended Citation}

Droubay, S. R., Nelson, T. S., Johnson, L. N., \& Ketring, S. A. (2004). Developing a program of research: A content analysis. ScholarsArchive. accepted for inclusion in Faculty Publications by an authorized administrator of BYU ScholarsArchive. For more information, please contact ellen_amatangelo@byu.edu. 


\title{
CAREER PATHS OF EMINENT RESEARCHERS IN MARRIAGE AND FAMILY THERAPY
}

\author{
Sarah R. Droubay \\ Thorana S. Nelson \\ Lee N. Johnson \\ Scott A. Ketring
}

\begin{abstract}
The mental health field of marriage and family therapy (MFT) needs more MFT researchers and more research to validate and verify the effectiveness and efficacy of MFT for the treatment of various mental health and relational problems. Knowing more about how successful researchers have developed their careers would be useful in both inspiring and guiding young MFT researchers toward success. Three eminent researchers whose careers have focused on MFT (James Alexander, John Gottman, and Howard Liddle) and their publications served as the research sample. Research questions addressing types of research, research samples, research questions, therapy model development, funding, and paths of the research careers were answered using content analysis. Results support guidelines that include having a focused research interest area, adhering to the scientist-practitioner model, obtaining funding, maximizing the utility of research samples, and generating publications.
\end{abstract}

KEY WORDS: marriage and family therapy; research and researchers; career paths; content analysis.

Sarah R. Droubay, MS, is at the Family Institute of Northern Utah, 965 S 100 W Ste 203 Logan, UT 84321. Thorana S. Nelson, PhD, is at Utah State University, 2700 Old Main Hall, Logan, UT 84322-2700 (thorana. nelson@usu. edu). Lee N. Johnson, PhD, is at The University of Georgia, Marriage and Family Therapy, Athens, GA 30605. Scott A. Ketring, $\mathrm{PhD}$, is in the Department of Human Development and Family Studies, Glanton House, Auburn University, Auburn, AL 36849-5604. Reprint requests should be sent to the second author. 
A large problem in the field of marriage and family therapy (MFT) today is the need for more research The field needs more evidence that MFT is a viable mental health modality for the treatment of a variety of problems, including diagnosable mental health disorders. In addition, there also is a need to better understand the process of MFT and what factors contribute to its effectiveness. Thus far, researchers have studied many phenomena related to therapy and the problems that bring people to therapy. Most research to date has been carried out by non-MFT trained researchers, often family psychologists. Notably, only five of the 27 authors in the recently published research update (Sprenkle, 2002) were trained as MFTs. Nonetheless, these accomplished researchers have furthered the profession of MFT and there are things MFTs can learn from them.

Crane, Wampler, Sprenkle, Sandberg, and Hovestadt (2002) suggest that there are several obstacles to training MFT researchers, including a culture that does not value research, a lack of role models within MFT, poor doctoral curricula in research, and research training that often is placed outside of the MFT programs Many MFTs (e.g., Julie Serovich, Sandra Stith, and Karen Wampler) are accomplished researchers and appear to be in the middle of accomplished careers. Although the field enjoys more MFT researchers than in the past, we need more and it would be useful to know how researchers in other disciplines who have long-standing careers developed those careers. Crane and colleagues suggest several improvements in master's and doctoral MFT programs to train students in the scientist-practitioner model of research, including MFT faculty mentors who are accomplished researchers. This article is an attempt to help in this endeavor by describing career paths possibly useful for MFT researchers, gleaned through investigating the research paths of three eminent researchers in MFT.

Although the MFT research field has produced successful researchers, there is no literature that is specific to either career paths in MFT research or what successful MFT researchers have in common. Understanding the ways that accomplished researchers in MFT have developed their research careers would assist both those who mentor MFT researchers and MFTs who may select research as a career path. Johnson (1991) pointed out that the literature thus far has not attended to how graduate students learn or are persuaded and encouraged to make research an integral part of their career paths. Additionally, Kremer and Bringle (1990) expressed concern that students without intense research experience run the risk of making hasty "uninformed" (p. 5) decisions to not pursue a 
research career, rather preferring a practice-oriented career path Descriptions of how a few eminent researchers have developed their careers may help inspire and direct new MFT researchers and their mentors.

The research that exists on career paths is quite limited. Perkins (2000) traced his own career path, highlighting the importance of integrating research, teaching, and community service work, presenting the possibility that a career path including (but not limited to) research may also be associated with success. Healy, Kosslyn, and Shiffrin (1992) edited a two-volume book as a tribute to their mentor, William Estes. These volumes were an interesting study in research career path development. It was clear that Estes was the authors' inspiration for developing their own careers.

Like the literature available on career paths, the literature available on career success is sparse. Career literature identifies internal (personality, self-efficacy, decision-making skills, family size, birth order, perceptions of early family life, interest in helping professions, and so on) and external (time, funding, facilities, students, mentors, recognition, and published reports and presentations) factors associated with success in a research career in general (e.g., Bakhai \& Halbreich, 1993; Bieschke, Bishop, \& Garcia, 1996; Bowden, 1997; Eiduson \& Beckman, 1973; Gordon \& Vicari, 1992; Halpain, Jeste, Katz, \& Lebowitz, 1997; Kremer \& Bringle, 1990; Perkins, 2000; Simonton, 1992; Trice, 1993). The existing literature also identifies some career development factors across the social sciences in general; what we do not know is how individuals develop their MFT research careers. The purpose of this article is to report on our research that identified external factors and trends in the research career paths of three successful and eminent researchers in marriage and family therapy-James Alexander, John Gottman, and Howard Liddle- - using their published works to begin building a model that may help guide MFT researchers and their mentors.

\section{METHOD}

\section{Population and Sample}

There were a number of important researchers who fit the definition of success and even eminence in that they were well known, well-published, well-funded, and well-respected. We chose Alexander, Gottman, and Liddle because of their research topics, not their academic degrees. We chose these researchers because they are well 
known, well respected, have sustained research careers in MFT, are clearly committed to MFT research, and have made connections to clinical work and the scientist-practitioner model.

The population for this study was all of the published works of Alexander, Gottman, and Liddle prior to 2000. A search using PsychINFO resulted in lists that totaled 209 publications for the three researchers. Alexander' list included 39 publications (one dissertation, one book, eight book chapters, and 29 journal articles). All were located as the sample for analysis. A total of 128 publications for Gottman were listed: one dissertation, eight books, 36 book chapters, and 83 journal articles. A total of 119 (93\%) were located for data analysis. A search for Liddle's publications resulted in a list of one dissertation, one book, ten book chapters, and 43 articles Fifty-one (93\%) of the 55 publications were located for analysis. A total of $94 \%$ of the indexed publications were located, resulting in sufficient, representative data to describe career paths through content analysis of publications.

We also obtained a rough count of the number of times the researchers' work was cited by their peers. Citations were counted manually from hard-bound copies of the Social Sciences Citation Index (SSCI). Alexander's name and therapy model (Functional Family Therapy; FFT) revealed 789 citations, Gottman's name revealed an astounding 3598 citations, and Liddle's name and therapy model (Multidimensional Family Therapy; MDFT) revealed 442 citations Gottman has developed a model that describes marital interaction and conflict, but had not developed a model of therapy at the time of the search. Liddle and Alexander both had developed named models of therapy (MDFT and FFT, respectively). It is probable that the count is conservative given the different ways that Gottman's name was spelled and the great number of citations that were hand-counted. The point remains, however: each of our researchers had been cited many, many times, adding to their stature as researchers.

\section{Procedure and Research Questions}

A coding sheet was developed to record data from each publication. To verify the utility, comprehensiveness, and reliability of the coding sheet, a random selection of three publications for each researcher were coded by two coders, resulting in confidence that the coding sheet would reliably serve the purposes of the research. Data were then coded by the first author. Questions that arose during the first coder's analyses were resolved through discussion between the first and second coder. 
Data for each research question were transferred from the coding sheets in chronological order onto seven lists (one for each research question). Keywords and phrases were highlighted in each publication. Data were summarized and entered onto the coding sheets. Phrases and keywords on the coding sheets were then highlighted for data analysis. Each list was used independently to examine the data for themes, patterns, and trends for each research question. We hoped to find patterns and themes that would assist researchers in the field of MFT to develop more focused, effective, and efficient research careers. The research questions for this project were:

1. What were the publication topics? How did topics change over time?

2. Did the researchers develop therapy models and how did they report the development?

3. How many publications credited a funding source?

4. What were the research samples? Did the samples change over time?

5. What types of research were conducted? How did they change over time?

6. What were the research questions? How did they change over time?

7. Did the researchers follow up on research ideas they generated?

\section{RESULTS}

\section{Publication Topics}

Data for this question were analyzed by looking for the purposes and themes stated in the titles, abstracts, and introductions of the publications. At times, the purpose was inferred from the text. Particular attention was paid to the development of research topics and changes in topic over time for each researcher.

Figure 1 diagrams Alexander's career path as it relates to publication topic. After receiving his $\mathrm{PhD}$, Alexander began investigating family therapy process, specifically, the dynamics of therapist-client variables and their effects on therapy outcome. It appears that he used the ideas from this research to develop FFT, a therapy model for intervening with families that include an adolescent who is involved with the legal system. FFT has been tested for effectiveness with 

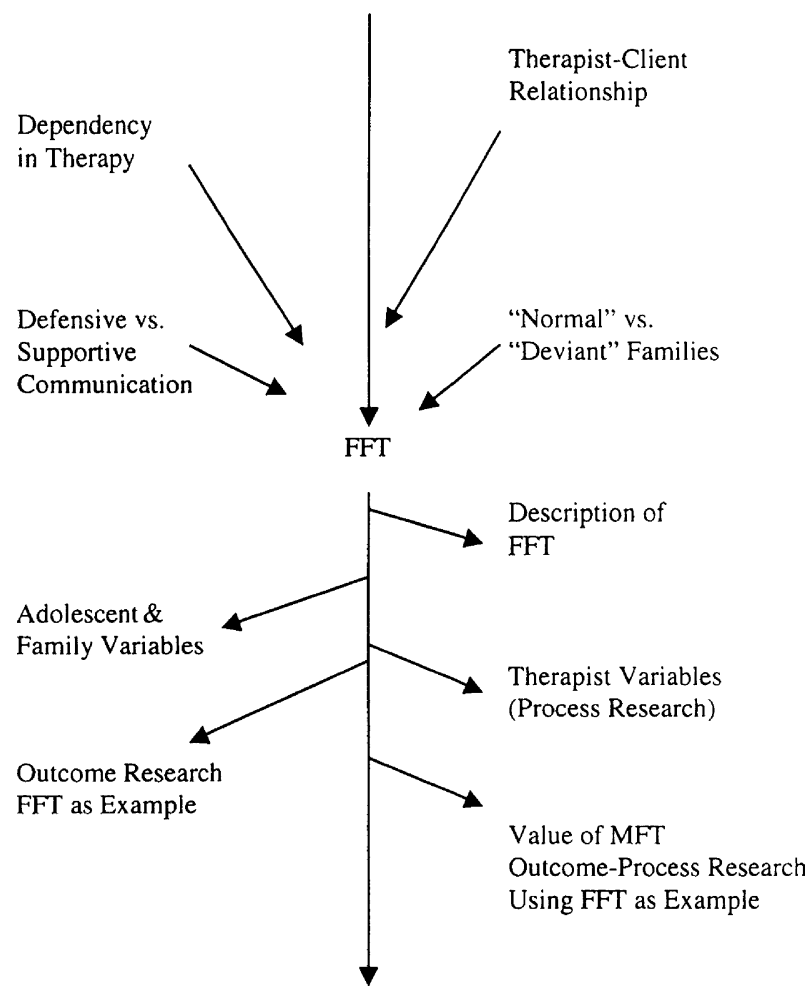

\section{FIGURE 1}

\section{Publication Topic Path: James Alexander}

various adolescent populations, focuses on specific concepts and interventions, and identifies family and therapy process variables that are most needed for positive treatment outcomes. Alexander also has written about family therapy research in general.

Figure 2 diagrams Gottman's publication topic path. He began his research career with methodology (time series analysis) and then used this method to isolate and/or identify moderating variables from within two different topic areas: the marital relationship (satisfaction vs. dissatisfaction, stability versus divorce, and conflict resolution) and children's friendships (relationship formation, popularity in school, social isolation, social and affect development of children, how children affect each other through language, and how friendship changes over time). The research on marital relationships and children's friendships began to overlap about 20 years into his research career, adding 


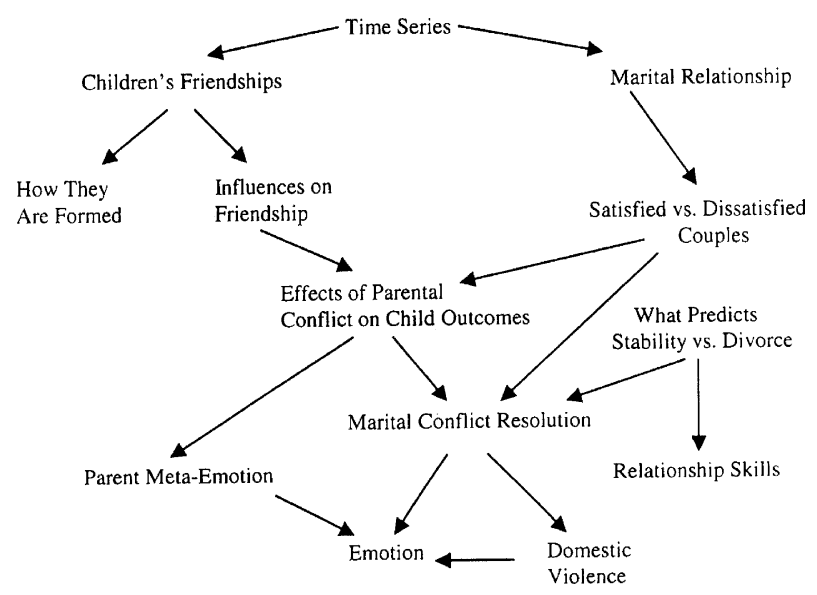

FIGURE 2

\section{Publication Topic Path: John Gottman}

a focus on the effects of parent conflict on children's friendships. This led to research on the role of emotion and meta-emotion in families and children. Gottman also extended his research into specific areas of marital interaction such as domestic violence.

Figure 3 illustrates Liddle's publication topic path. Liddle began by publishing his ideas on family therapy supervision and training. While most of this work was oriented in systems theory and consisted of "think" pieces, some was based on surveys of students, trainees, and supervisors. Later, Liddle published a model of family therapy for treating substance abusing adolescents and their families, focusing on drawing interesting parallels between the development of the therapist-client relationship and the process of therapy over time. He also expanded his work to therapy with delinquent adolescents in general.

\section{Model Development}

This research question explored (a) whether or not each researcher named a model of therapy or of family interaction, (b) whether or not each researcher developed such a model without naming it, and (c) how each researcher treated the therapy model or model in general: describing it, developing its concepts, validating an intervention, and/or testing it on new populations or with new refinements based on previous research. Particular attention was paid to the way the model evolved over time. 


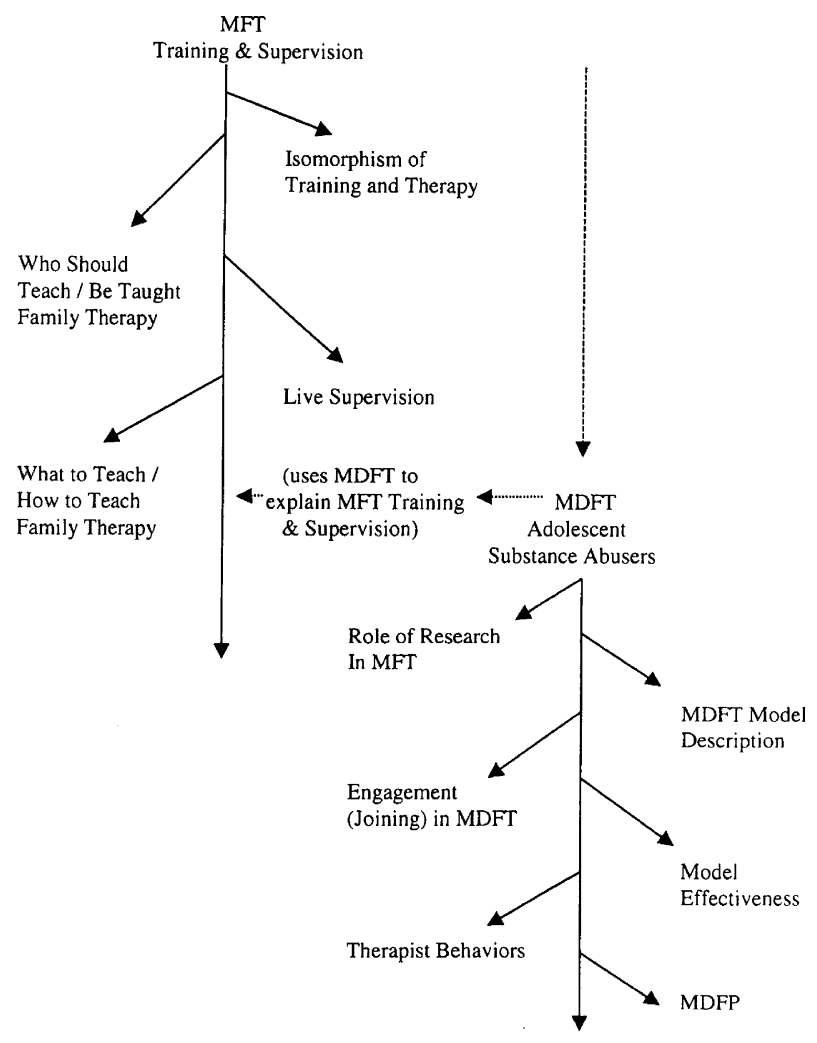

FIGURE 3

Publication Topic Path: Howard Liddle

Alexander. Twenty-five (64\%) of Alexander's publications were related to the development and description of FFT. He began by looking at specific therapy processes (client-therapist interaction) and then described his model of therapy, highlighting specific parts of the model. Thirty-three (85\%) publications reported on building the model. Alexander appeared to focus on refining or expanding specific components of the therapy model by using process research to understand whether interventions from the model were effective and worked as intended. Seven publications validated specific interventions of FFT, testing the therapy model on samples of families with troubled adolescents. Thus, FFT seems to be based on both theory (like Liddle) and research (like Gottman). Six of the publications applied FFT to other 
populations. Alexander appeared to focus on a matching-to-sample philosophy of developing interventions specific to particular populations. He worked with other investigators and in other modalities (e.g., community interventions), perhaps recognizing that one therapy model may not be effective with all clinical problems.

Gottman. A total of 89 (75\%) of Gottman's 119 publications described a theoretical model of marital interaction, children's friendships, or methodology. A total of 98 (82\%) publications went beyond description, building the model through expansion, extension, and refinement. Clearly, this represented the bulk of Gottman's research. A pattern emerged: Gottman used the time-series methodology he developed to investigate components of his model of marital interaction, for example. Moderating variables identified by the research were teased out and examined independently to identify their roles and functions. Ten longitudinal studies and other publications that validated interventions looked specifically at the accuracy of using these moderating variables, such as those that predicted marital stability. Twenty-five publications applied an existing theory to a new population, such as those that applied time-series methodology to new populations. Since this study was conducted, Gottman has developed a model of therapy.

Liddle. Of a total of 51 located publications, 46 (90\%) of Liddle's work was coded as describing the development of his models for supervision of therapy or MDFT. Most of these articles describe MDFT, which was based on his ideas of systems therapy with families with troubled adolescents. A total of 37 (73\%) publications expanded, extended, or refined theory in some way, thus building the model. Four publications validated an intervention for MDFT. Liddle also looked at specific interventions that he included in MDFT, testing the therapy model with families of adolescents and verifying it in outcome and process studies. Four publications applied one of his models to another population.

\section{Funding Sources}

This question sought to understand the nature of external funding that each researcher used for publications. Because data regarding funding were from published resources only, it is not possible to know amounts of funding or to know about the researchers' other resources (e.g., university or funding that is not typically reported with 
publications). Alexander's funding was split mostly between National Institutes of Drug Abuse (NIDA) and National Institute of Mental Health (NIMH).

Of Alexander's 39 publications, $24(62 \%)$ cited funding that was mostly split between NIDA and NIMH. Identification of the Principal Investigator (PI) was not always clear. At least 12 of the 24 funded publications were solely or partially awarded to Alexander. Alexander cited 12 separate awards.

Of Gottman's 119 coded publications, $73(61 \%)$ cited funding sources. The main funding source was NIMH, including the prestigious Research Scientist Award, which was cited in 38 books, book chapters, and journal articles. Gottman appeared as PI on 27 (23\%) of the other publications; a total of $62(51 \%)$ cited Gottman as PI or coinvestigator. The publications for which he appeared as PI (27 publications) reflect a combination of 13 separate awards. In total, Gottman cited 34 separate awards.

Of Liddle's 51 coded publications, funding was cited for 21 (41\%), mostly from NIDA and naming Liddle as PI. One article cited funding to another researcher solely. A total of 20 of the publications cited funding to Liddle as PI or co-investigator. A total of 16 funding sources were cited.

\section{Research Samples}

Alexander used clinical samples of adolescents involved in the juvenile justice system and their families. Much of his research with this population also included "normal" adolescents and their families as a comparison group. His process research used samples of therapist and client behaviors from videotapes. The type of research sample used by Alexander did not appear to change over time.

Gottman used non-clinical families and subsystems of families including children, married couples, and parent-child systems. He used the data sets for several analyses and also conducted longitudinal studies on single samples. His research samples did change with research topic, but consistently consisted of families and subsets of families.

Liddle's research samples included family therapy training and supervision publications, family therapy programs, and family therapy efficacy studies. Later, as his research topic shifted to MDFT, he used clinical samples of adolescents and their families dealing with drug abuse, as well as videotapes of therapists and clients in session. 


\section{Types of Research}

This question addressed several aspects of research. First, research publications were defined as those that discuss samples, data, and analyses and we wondered how many of each researcher's publications dealt specifically with research defined this way. Second, we attempted to determine whether the research was exploratory in nature, whether it extended the research to new variables or new combinations of variables, or whether it refined the research by testing a model on a new population. Finally, we examined the research to determine whether it tested an idea against an outcome-clinical or not, investigated particular processes in the phenomenon, or tested a particular process against a particular outcome.

Twenty-one (54\%) of Alexander's 39 publications included information derived from data analysis. All of this research included clinical samples of delinquent adolescents and their families. Initially, the research was exploratory and related to outcome, while also including process research looking at therapy components such as the clienttherapist relationship. Later, Alexander focused on new applications of the model and fine tuned understandings of model components and compared these to outcomes.

Sixty-four (54\%) of Gottman's 119 coded publications fit the definition of research. Gottman began with human, non-clinical samples (children's friendships and marital dyads) and exploratory research. He continued studying these populations and extended the research to isolate specific variables, for example, physiological variables such as heart rate changes in male perpetrators of domestic violence during conflict conversations and how parental vagal tone (condition of the vagus nerve, which affects the limbic system functions of mood, motivation, and alertness, among others) may or may not serve as a protective factor to buffer children from the harmful effects of marital hostility. He also conducted research examining which variables predict divorce. Gottman developed and tested new environments for study (having couples stay overnight in an apartment-like laboratory) and new measurements ("talk tables" where couples rated their own as well as their partners' intention of communication), refining what he had previously learned. From the research on processes of marital dynamics, Gottman made recommendations for clinicians. Very little of the research examined interventions in clinical populations per se. One example was a study (Glass, Gottman, \& Shmurak, 1976) that compared teaching dating skills with the use of cognitive 
self-statements to identify which would be more effective in improving real-life dating situations.

Thirteen (25\%) of Liddle's 51 coded publications fit the definition of research. Liddle initially conducted research on theory development, including exploratory research that studied variables of marriage and family therapy training and supervision. Although much of this work involved reviews of literature and the integration of theory and his ideas with the literature, he also surveyed trainers, trainees, and supervisors. Later, Liddle's clinical research included developing a model of therapy (MDFT) and testing its efficacy through process, outcome, and process-outcome research with families of substance abusing adolescents. In addition to this clinical outcome research, Liddle extended his research to study specific aspects of the MDFT, such as how therapists and clients respond during therapeutic impasses. He then used this information to refine the therapy model and test its application by examining how therapists responded with a "shift event" when an impasse in therapy was reached.

\section{How Did the Research Questions Change Over Time?}

We were curious about the researchers' questions over time. Did they start with something, refine it, and then extend it? Did they move from topic to topic? Did they stop researching certain topics or questions and start researching others? Were they focused?

Alexander's research questions sought to develop, refine, and test FFT in multiple ways. Initially, research questions investigated the treatment model and specific parts of the model. Other questions served to build the therapy model. He also conducted process and process-outcome research that identified therapist behaviors that contribute to positive outcomes. Thus, his questions identified important aspects of FFT and then tested them over time without deviating from FFT as his focus.

Gottman's research questions had a definite trend and seemed focused on developing a complete understanding of the phenomena he was studying, whether it was marital conflict, children's friendships, or the relationship between the two. For example, he investigated particular variables, relationships among variables, and the predictability of certain variables toward divorce. He even studied physiological phenomena using equipment to measure heart rate during marital conflict, testing the validity of the measuring tool as well as the relationship of the particular variable to the phenomenon. Gottman's research was primarily exploratory and he seldom posed 
specific hypotheses in the articles. Research questions changed over time in terms of the research topic, yet were consistent in that they served to better understand the phenomenon and then test the theory.

In Liddle's clinical research, the questions he posed served first to build and strengthen his model of therapy. Later, he asked process oriented questions to identify particular therapy variables that contribute to positive outcomes in therapy, to ascertain whether therapists actually were using the model as prescribed, to identify interventions that contributed to the client-therapist alliance, and to test the efficacy of the therapy model. Questions were different over time in that his research focus changed from understanding MFT training and supervision to the development of MDFT. However, his questions within each topic were focused and built on previous research.

\section{Follow-up Research}

The last research question addressed (a) how often the researchers made recommendations for further research, and (b) whether they followed up on these suggestions themselves.

A total of 20 (51\%) of Alexander's publications made recommendations about future research. Alexander personally followed up on very few of these recommendations, with two notable exceptions. One was his study measuring the effects of negative and positive reframing on defensive communication. An earlier article had suggested that future research examine how stress associated with a particular task in therapy affects subsequent behaviors. Alexander also followed up on his suggestions for studies of the effects of therapist gender, extending it to include interactions related to client gender. He also made general recommendations for researchers and research, training of FFT clinicians, and suggested the need for more outcome-based clinical research, which he, himself, continued to do.

Gottman tended to followup on the research ideas he generated in $80(67 \%)$ of his coded publications. For example, in one of his publications, he recommended that future research identify potential buffers for children exposed to marital stress or conflict. Later studies not only examined child buffers, but family and parental buffers as well. It appeared that perhaps the questions he was likely to propose pointed him in new directions for researching the particular phenomenon. Additionally, he made recommendations for other researchers and clinicians, perhaps setting limits for his own research trajectory and encouraging others to continue. He did not limit his recommendations 
to research-oriented publications, as evidenced by the finding that only 64 of his publications were research-oriented, yet 80 contained some form of recommendation for future research.

Twenty-five of Liddle's 51coded publications suggested future research, including publications that were not research-oriented themselves. He followed up on only a few of these suggestions himself. He also made general calls for research such as the need for more MFT research that could be applied to clinical practice. Finally, he made suggestions for the field that went beyond research: recommendations for future researchers; integration of theory, research, and practice; and the need for the field of MFT to examine itself systemically alongside other mental health fields.

\section{DISCUSSION}

The goal of this research was to study the career paths of three accomplished researchers of marriage and family therapy to provide examples for future MFT researchers and their mentors. It is clear that our selected researchers were all prolific. Alexander, with the fewest numbers of publications during his career up to 2000, still had many more publications than the "average" of nine for eminent psychologists reported by Simonton (1992). It also is clear that these three researchers' work is cited with great frequency. Searches revealed over 3500 citations of Gottman's work, 789 citations of Alexander's work, and 442 citations of Liddle's work, it is apparent that these authors' publication topics are of high interest to others.

All three researchers also focused their attention on at least one sustained topic. Liddle's topics of interest were consistent within each phase of his publication career, first with MFT training and supervision and then with development of the MDFT therapy model. Alexander consistently focused on FFT, first developing then refining and applying the therapy model to additional populations. Gottman consistently studied three main topics: marital interaction, children's friendships, and methodology. He also focused on different processes and parts of those interest areas. Additionally, all three researchers appeared to have integrated theory, research, and practice.

All three researchers received considerable funding that supported their research and writing. It is not possible to know from the literature itself what the grant-writing and funding patterns were for each, but it is clear that they used NIMH and NIDA funding as well as other sources of funding to support their work. They also acted 
responsibly to these funding sources by extending the research, developing research programs, and informing other researchers and clinicians about many aspects of the research and its potential applications. For example, Gottman used his Research Scientist Award (K award) to great advantage to develop his research program. All three researchers used multiple funding sources and multiple grants to finance their work. Thus, up and coming researchers would do well to integrate research, theory, and practice, and to develop skills in securing funding.

It is evident from their publications that all three researchers used research samples that fit the topic being studied. However, each used the samples in different ways. For example, Gottman analyzed data from at least five different samples in multiple ways. Alexander analyzed data from three samples in multiple ways as well (including one longitudinal study), but subsequent analyses resulted in only a handful of additional publications. Unlike Alexander and Gottman, Liddle did not use research samples (either longitudinally or through additional analyses of data) more than once, according to the information available from the publications.

One particularly valuable finding is the description developed that illustrates the consistent focus for each researcher on the type(s) of research they conducted throughout their research career path. Alexander's research began with a clinical population-juvenile delinquents-and he began to build a model of treatment. He conducted exploratory and theoretical research to develop a model of therapy and the necessary parts of the model. He integrated clinical research (process, outcome, and process-outcome) with exploratory and theoretical research to refine and extend the therapy model, variables of the therapy model, and measurements of behaviors addressed by the therapy model.

Gottman began with nonclinical populations and conducted mostly exploratory research, seeking to understand the phenomena, to distinguish parts of the theoretical model, or to refine relationships between parts of the model and theoretical research, defining, refining, and building the model.

Liddle began with theoretical research, presenting, building, and refining his ideas about MFT training and supervision and then conducted research that explored and defined the process of MFT training and supervision. When he shifted to research using MDFT, he began conducting clinical research with the population of troubled adolescents with substance abuse problems as well as theoretical research, using his ideas about family therapy to build his model of therapy. $\mathrm{He}$ 
then tested and refined the model with process, outcome, and processoutcome research. A consistent focus of research topics is common among these three researchers.

All three researchers were likely to make different kinds of recommendations for further investigation. Liddle tended to make recommendations for the field in general and called for more outcomerelated research. Alexander tended to make recommendations for the training of FFT and for more process research. This trend was not true of Gottman's research in which his recommendations tended to be "sneak previews" of research that most likely was already being planned and/or conducted.

\section{Limitations}

This project was exploratory and did not test any hypotheses about what goes into the career path of a successful MFT researcher. Therefore, conclusions should be taken with caution. Also, a major limitation is that no female researchers were studied. In order to make career decisions, it would be good for women to have more information about the similarities and differences from men they might find in their career paths. Also, only three researchers' work was examined. It is probable that other successful researchers' work would reveal different patterns.

More information could reveal different and/or more detailed pictures of the career paths of these men. However, given the volume of data and the success with which the publications were found, we believe that the picture presented here is a fair one. Interviews and examination of curriculum vitae would help illuminate this issue, as might analysis of details of other work such as professional presentations.

\section{Recommendations}

The information from this study, we hope, will be of great interest to those who are learning to conduct research and to their teachers. Those who are teaching research will benefit by understanding and relaying to students the importance of being conscious and purposeful about plans for their careers, rather than simply going from topic to topic or funding source to funding source as opportunities arise. Young researchers might select a path from these three that makes most sense to them rather than testing several topics before focusing on one. It appears from these data that direction and focus are critical. A quote 
by Gottman illustrates that one can begin a successful research career by seeking greater understanding of an area of interest that is of personal relevance (Gottman, 1990):

When I began teaching, my interests were not substantive but methodological. My advisor, Richard McFall, suggested that I select a research problem of some interest to me rather than sticking with primarily methodological and statistical questions. After a lot of soul searching, I decided that I would study marriage, and social isolation in children. Frankly, I selected these two areas of inquiry because they had been the source of considerable pain in my own personal life. (p. 249)

It seems that successful professional preparation comes from selecting a topic of interest and once that topic has been selected there are several ways to go about conducting research on the topic. However, this research has highlighted several important considerations. (1) Research training is necessary; all of these researchers had knowledge of research methods. (2) The importance of the scientist-practitioner model was evident as these researchers consistently integrated research, therapy, and, where applicable, clinical practice. (3) One commonality among the three researchers is the consistent focus of their research at any one time. (4) Once researchers have skills in sound research methodology and can integrate theory and practice with those skills, they need to develop skills in securing external funding.

\section{CONCLUSION}

This project analyzed data from the publications of three eminent researchers to learn more about the career paths of successful researchers in marriage and family therapy. Data suggest that successful researchers in MFT produce many publications, focus clearly on sustained research topics, develop their model(s) as well as its components, contribute this to other studies and publications, obtain funding for their work, maximize the utility of their research samples, and contribute to the field through their recommendations. They begin with ideas, examine related phenomena using exploratory methods, develop models to both explain and understand phenomena or to intervene in certain problems, examine the processes of the phenomena in detail, demonstrate the effectiveness of their interventions and treatments, and generate new ideas. Although we studied the career paths of researchers who were not trained in MFT programs, we 
believe that their examples may provide models for MFT research. It is our hope that young MFT researchers and their mentors will learn from these data in ways that will allow them to be more focused and efficient, adding to the body of knowledge of marriage and family therapy and to the numbers of researchers who succeed in doing research.

\section{REFERENCES}

Bakhai, Y., \& Halbreich, U. (1993). Development of junior faculty in resource-poor departments of psychiatry. Academic Psychiatry, 17(2), 84-90.

Bieschke, K. J. , Bishop, R. M., \& Garcia, V. L. (1996). The utility of the Research SelfEfficacy Scale. Journal of Career Assessment, 4(1), 59-75.

Bowden, V. (1997). The career states system model: A new approach to analyzing careers. British Journal of Guidance and Counseling, 25(4), 473-490.

Crane, D. R., Wampler, K. S., Sprenkle, D. H., Sandberg, J.G., \& Hovestadt, A. J. (2002). The scientist-practitioner model in marriage and family therapy doctoral programs Journal of Marital and Family Therapy, 28, 75-83.

Eiduson, B. T., \& Beckman, L. (1973). Science as a career choice: Theoretical and empirical studies. New York: Russell Sage Foundation.

Glass, C., Gottman, J., \& Shmurak, S. (1976). Response-acquisition and cognitive selfstatement modification approaches to dating-skills training. Journal of Counseling Psychology, 23(6), 520-526.

Gordon, R. A., \& Vicari, P. J. (1992). Eminence in social psychology: A comparison of textbook citation, Social Sciences Citation Index, and research productivity rankings Personality and Social Psychology Bulletin, 18(1), 26-38.

Gottman, J. M. (1990). Finding the laws of close personal relationships. In I. E. Siegel \& H. H. Brody (Eds.), Methods of family research: Biographies of research projects, Vol 1: Normal families (pp. 249-263). Hillsdale, NJ: Erlbaum

Halpain, M. C., Jeste, D. V., Katz, I. R., \& Lebowitz, B. D. (1997). The first summer research institute in geriatric psychiatry. American Journal of Geriatric Psychiatry, 5(3), 238-246.

Healy, A. F., Kosslyn, S. M., \& Shiffrin, R. M. (1992). Essays in honor of William K Estes, Vol. 2: From learning processes to cognitive processes Hillsdale, NJ: Erlbaum.

Johnson, D. C. (1991). Persuasion: A process in the training of counselors. Contemporary Social Psychology, 15(1), 12-15.

Kremer, J. F. , \& Bringle, R. G. (1990). The effects of an intensive research experience on the careers of talented undergraduates. Journal of Research and Development in Education, 24(1), 1-5.

Perkins, D. D. (2000). Research, teaching, and service in applied, multidisciplinary academic programs and in community organizations. Journal of Prevention and Intervention in the Community, 19(2), 121-128.

Simonton, D. K. (1992). Leaders of American psychology, 1879-1967: Career development, creative output, and professional achievement. Journal of Personality and Social Psychology, 62(1), 5-17.

Sprenkle, D. H. (2002). Effectiveness research in marriage and family therapy. Alexandria, VA: American Association for Marriage and Family Therapy.

Trice, A. (1993). Factors influencing sustained research careers among experimentally trained psychologists. Psychology: A Journal of Human Behavior, 30(3-4), 24-26. 
Copyright of Contemporary Family Therapy: An International Journal is the property of Kluwer Academic Publishing and its content may not be copied or emailed to multiple sites or posted to a listserv without the copyright holder's express written permission. However, users may print, download, or email articles for individual use. 